\title{
Ideología y reconocimiento de los pueblos indígenas: análisis del discurso político del Consejo Regional Indígena del Cauca (CRIC)*
}

\author{
HARVEY HUMBERTO MERCHÁN CANTE*
}

jmerchan123@gmail.com

Recepción: 18 de noviembre de 2014

Aprobación: 11 de diciembre de 2014

Forma de citar este artículo: Merchán Cante, H. (2015). Ideología y reconocimiento de los pueblos indígenas: análisis del discurso político del Consejo Regional Indígena del Cauca (CRIC). Cuadernos de Lingüística Hispánica, 26, 139-155, Tunja: Uptc.

* $\quad$ Este artículo de investigación muestra resultados preliminares de un trabajo titulado "Análisis del discurso político del CRIC. Ideología y reconocimiento de los pueblos indígenas del Cauca".

** Estudiante de la Maestría en linguística, Universidad Pedagógica y Tecnológica de Colombia. 


\title{
Resumen
}

En este artículo se muestra, desde la perspectiva del Análisis del discurso, cómo la comunidad indígena del Cauca construye su discurso sobre inclusión; en particular, cómo esta colectividad representa socialmente a los integrantes del conflicto colombiano y cómo legitima y justifica sus políticas, sus opiniones y actitudes hacia la realidad de nuestro país. Asimismo, se indaga sobre cómo la ideología condiciona y determina su discurso social. El propósito de estos análisis es proporcionar las herramientas necesarias para valorar la participación de este grupo étnico sobre la formación de técnicas que pueden caracterizar su propuesta y su ideología. Para ello, se aborda un análisis del discurso que se centra en las macro-estrategias semánticas y micro-estrategias léxico-semánticas que se desprenden del discurso del Consejo Regional Indígena del Cauca.

Palabras clave: Consejo Regional Indígena del Cauca, Análisis del discurso, legitimación, inclusión, ideología, representación social, política.

\section{Ideology and inclusion of indigenous peoples: political discourse analysis of the indigenous regional council of Cauca.}

\begin{abstract}
This article presents the manner in which the indigenous community of Cauca constructs its discourse from the scope of discourse analysis; particularly, how this community socially represents the actors of the Colombian conflict and how it legitimates and justifies its policies, opinions and attitudes towards the reality of our country. Likewise, inquiries into the conditioning and determining roles of ideologies in their social discourse are made. The purpose of these analysis is to provide the necessary tools for recognizing the participation of this ethnic group in the development of techniques to characterize their proposal and their ideology. To that end, a discourse analysis is carried out, based on the semantic macro strategies and lexical semantic micro strategies found in the discourse of the indigenous regional council of Cauca.
\end{abstract}

Key words: Indigenous regional council of Cauca, discourse analysis, legitimation, inclusion, ideology, social representation, politics. 


\section{Idéologie et inclusion des peuples indigènes: analyse du discours politique du Conseil Régional Indigène du Cauca}

\section{Résumé}

Dans cet article on montre, dès la perspective de l'Analyse du discours, comment la communauté indigène du Cauca, construit son discours sur l'inclusion; en particulier, comment cette collectivité-ci représente socialement les membres du conflit colombien et comment elle légitime et justifie ses politiques, ses opinions et ses attitudes vers la réalité de notre pays. De la même manière, on enquête sur comment l'idéologie conditionne et détermine son discours social. Le propos de ces analyses est celui de proportionner les outils nécessaires pour mettre en valeur la participation de ce groupe ethnique sur la formation de techniques qui puissent caractériser sa proposition et son idéologie. Pour cela, on aborde une analyse qui se centre sur les macro-stratégies sémantiques et microstratégies lexico-sémantiques qui se dégagent du discours du Conseil Régional Indigène du Cauca.

Mots clés: Conseil Régional Indigène du Cauca, Analyse du discours, légitimation, inclusion, idéologie, représentation sociale, politique.

\section{Ideologia e inclusão dos povos indígenas: análise do discurso político do Conselho Regional Indígena de Cauca}

\section{Resumo}

Neste artigo mostra-se, desde a perspectiva da Análise do discurso, como a comunidade indígena de Cauca constrói seu discurso sobre inclusão; em particular, como esta coletividade representa socialmente aos integrantes do conflito colombiano e como legitima e justifica suas políticas, suas opiniões e atitudes para a realidade de nosso país. Assim mesmo, se indaga sobre como a ideologia condiciona e determina seu discurso social. 0 propósito desta análise é proporcionar as ferramentas necessárias para valorizar a participação deste grupo étnico sobre a formação de técnicas que podem caracterizar sua proposta e sua ideologia. Para isso, se aborda uma análise do discurso que se centra nas macro estratégias semânticas e micro estratégias léxico-semânticas que se desprendem do discurso do Conselho Regional Indígena de Cauca.

Palavras chave: Conselho Regional Indígena de Cauca, Análise do discurso, legitimação, inclusão, ideologia, representação social, política. 


\section{Introducción}

La legitimización de las luchas indígenas y el proceso de vinculación en las estructuras políticas organizativas colombianas, se ha originado inicialmente en el departamento del Cauca. Este se fundamenta en las normas naturales y la relación con los seres de la tierra y el territorio como principio de toda vida. Establecidos en estos mandatos materiales-espirituales se reclama respeto, apoyo y garantía al ejercicio pleno de la libre autodeterminación en la búsqueda de la permanencia según sus costumbres, como pueblos originarios de América.

Para los gobiernos, estos principios ancestrales no son suficientes ni válidos. Se les ha invisibilizado las normas jurídicas nacionales e internacionales que llaman a garantizar lo que siempre ha precedido como pueblos milenarios. Fortificados en estos fundamentos, se exige el derecho a la tierra, a un territorio libre, a vivir en equilibrio con la naturaleza y a desarrollarse de manera autónoma tal y como lo consagra la constitución política colombiana de 1991. Desde la perspectiva anterior, se expondrá las herramientas discursivas más comunes en su discurso político que ayudan a legitimar y justificar sus políticas, sus opiniones y actitudes hacia la realidad colombiana.

Para este fin, se llevará cabo un análisis a partir de las estructuras macrosemánticas de los distintos discursos escogidos (corpus), luego se determinarán las micro estrategias léxico semánticas más recurrentes, para mostrar en qué medida estas influyen en su caracterización discursiva.

Se ha escogido como tema para este artículo el análisis del discurso para exponer la medida en que este incidió para la inclusión indígena en la constitución colombiana de 1991 y las estrategias lingüísticas utilizadas para la exposición de sus argumentos. Se enfatizarán aquellas destrezas que particularizan el discurso del CRIC ${ }^{1}$, que influyen en la creación de una política de inclusión para los pueblos indígenas de Colombia.

1 CRIC: Consejo Regional Indígena del Cauca. 


\section{Poder y análisis discursivo}

En el mundo actual se vive en una constante indecisión precisamente porque no existe una aproximación certera acerca de las diversas formas en las que el discurso se puede contextualizar, varios de estos son los que se pronuncian en la educación, la ciencia, la economía, los medios de comunicación y la política. Aunque este último, generalmente, establece la preferencia ante los demás. Lo poco que se conoce de estos discursos, a simple vista es su normatividad en el uso del lenguaje.

A raíz de esto, uno de los filósofos más reconocidos de nuestra época, Michel Foucault (1970), considera que el discurso no debe mantenerse en el "tabú del objeto, ritual de la circunstancia, derecho exclusivo o privilegiado del sujeto de habla," ${ }^{2}$ que irrumpe la particularidad discursiva. Con este planteamiento, Foucault abre una puerta a la pluralidad del discurso, esto significa que el discurso no tiene un orden preestablecido para manifestarse, sino que se descontinuó, ello no implica equivocidad discursiva, ya que permite recoger diversas ideas sobre el discurso como una práctica dispersa en la vida concreta.

Partiendo de la perspectiva en la cual la producción material en toda sociedad, inclusive en los propios discursos, está controlada, seleccionada y redistribuida por cierto flujo de normatividades, Foucault distingue los siguientes tipos de estos procedimientos: en primer lugar, los procedimientos de exclusión (carácter externo al propio discurso), entre los que se destacan los de prohibición. ${ }^{3}$ En la política, convirtiendo al mismo discurso en objeto de la lucha política y no en su mera manifestación; los de separación y rechazo, de la separación entre razón y locura; y los de oposición entre lo verdadero y lo falso, que son los que a través de su propia historia han dado su forma a nuestro conocimiento general, a la distinción entre lo que es conocimiento y lo que no, discernimiento.

En distinta medida, se hace un acercamiento desde el discurso al estigma del poder, Foucault, al igual que otros filósofos y analistas del contenido discursivo en distintos contextos sociales, crea una relación directa en lo que a discurso y poder se refiere. Este análisis discursivo sobre la dicotomía discurso y poder ha sido llevado al punto máximo de estudio por distintos investigadores como Teun Van Dijk (2009), como Foucault, establece una relación crítica sobre el uso del discurso como manipulación de masas, pero ya más profundamente como análisis crítico del discurso, establece otro tipo de normatividad.

\footnotetext{
2 Tomado de http://michelfoucault1.blogspot.com/2010_10_01_archive.html

3 Hace relación a esto en su libro "El orden del discurso" en donde pone énfasis en los procedimientos de exclusión en el discurso
} 


\subsection{Análisis crítico del discurso}

El Análisis Crítico del Discurso (ACD) es una perspectiva crítica que se enmarca dentro de la disciplina general del análisis del discurso. Como paso preliminar, conviene señalar algunas teorías sobre lo que se entiende por discurso. Según Van Dijk (2009), "el discurso se define como un fenómeno -multidimensional- muy complejo y estructurado que engloba dimensiones lingüísticas, interaccionales, prácticas sociales, representaciones mentales, etc." Para poder definir el discurso, es necesario atender a estas (y otras) dimensiones que lo componen, condicionan e interactúan con él de algún modo.

Teniendo en cuenta este postulado, podemos considerar el discurso como una práctica social en la que se expresa y comunica una interpretación de la realidad que va cambiando en función de cómo el discurso se construye. El análisis crítico del Discurso, por lo tanto, tiene que ver con el cuestionamiento activo de los supuestos que conforman una construcción social.

\subsection{Discurso político}

Dado que el discurso político supone una de las formas más directas de hacer política y de crear opinión, el estudio del discurso de los grupos indígenas de Colombia, a partir de su discurso político antes de la constitución colombiana del 91, nos revelará no solo cómo se articula y se legitima las políticas indígenas, sino también las actitudes, los prejuicios y estereotipos que se proyectan hacia la representación social que se hace de la misma, y cómo la ideología del CRIC trabaja en la conformación de esta representación. De este modo, podremos evaluar qué papel juega el CRIC sobre la formación política e ideológica del pueblo colombiano.

La teoría de Teun Van Dijk acerca del ACD, es una propuesta metodológica y teórica, en tanto se alimenta de categorías como "ideología", "poder", "hegemonía". En este trabajo, el ACD resulta ser un instrumento metodológico, para el tratamiento del discurso recogido, además de diversas fuentes que permiten entender la forma en que las clases en el poder representan al indígena en Colombia.

El análisis de los archivos y documentos así como los comunicados de prensa elaborados por las organizaciones indígenas (principalmente del CRIC), a través del ACD ha permitido entender la estructura narrativa de cada texto; cabe recordar que de estos documentos fue seleccionado el discurso del representante indígena a la constitución de 1991, Alfonso Peña Chepe, por su extenso contenido semántico y contextual. De esta

4 En Discurso y poder Van Dijk hace referencia directa a esta tríada como parte del discurso político. 
manera, es posible dar cuenta del lugar desde el cual se representa lo indígena en el escenario político. Se intenta explicar, tanto desde la perspectiva de las clases en el poder, como desde las organizaciones indígenas, el contexto social y las tensiones políticas alrededor del movimiento indígena en Colombia.

De la misma forma, la ideología se encuentra demarcada en los distintos discursos de las comunidades indígenas, pero también, en los discursos políticos construidos para referirse al "problema" de lo indígena en Colombia.

Es aquí en donde los diversos discursos referidos a las comunidades indígenas de nuestro país, se inclinan hacia el racismo y exclusión de los pueblos autóctonos del territorio nacional; como lo dice Van Dijk (2003), "El racismo proporciona la estructura explicativa fundamental de la innegable experiencia y observación cotidianas", también menciona al respecto "[...] que más blanco significa "mejor” y (más) negro o (más) indígena significa "peor" sea cual sea el ámbito social y el tipo de experiencia” (p. 111).

El discurso indígena en Colombia se entiende a raíz del significado que toma a partir del contexto social en que se enmarca nuestro país. De esta forma, estos discursos, en las diferentes estancias políticas, no deben entenderse como simples discursos de dignidad y hegemonía racial, sino, por el contrario, debe entenderse a partir de las múltiples relaciones de poder político entre los gobernantes y dirigentes, incluyendo también a los diferentes partidos políticos colombianos.

\section{Metodología}

Para hacer un análisis coherente se utilizó la división temática y macroproposicional. Cada macro proposición se deriva de una serie de microproposiciones concretas en el texto. Esta división temática obedece al hecho de que, dependiendo de los distintos temas tratados, la argumentación y la retórica toman unas características diferentes, ya que el contenido semántico de cada uno de los temas determina el contenido y la forma de los argumentos utilizados.

En consecuencia, fue necesario analizar de forma separada cada una de las macroproposiciones, de manera que puedan apreciarse estas características como paso anterior, para establecer posibles estándares sobre el uso de las estrategias retóricoargumentativas más frecuentemente utilizadas por el CRIC.

5 Entiéndase que algunos sectores políticos colombianos buscan hacer ver a las comunidades indígenas como un problema para el avance social. Declaración del CRIC a los 40 años de su constitución, La María, febrero de 2011. 
Conjuntamente con este análisis cuantitativo, se realizó un análisis cualitativo para mostrar cómo las distintas estrategias se relacionan entre sí en cada macroproposición y para comprender cómo se articulan la legitimación y justificación de las acciones políticas, las actitudes y las opiniones de la comunidad Páez. Así, el análisis cualitativo y cuantitativo, nos permitió establecer patrones en el uso de las distintas estrategias retóricoargumentativas empleadas.

En el análisis hecho a los comunicados de prensa del CRIC, sumándolo al discurso del constituyente Alfonso Peña Chepe, se pudo obtener:

\begin{tabular}{|c|c|c|c|c|c|c|c|c|c|}
\hline $\begin{array}{l}\text { Fin del } \\
\text { Conflicto }\end{array}$ & $\%$ & Reconocimiento & $\%$ & $\begin{array}{l}\text { Defensa de la } \\
\text { Autonomía, } \\
\text { Cultura y } \\
\text { Territorio }\end{array}$ & $\%$ & $\begin{array}{c}\text { Manejo del } \\
\text { Estado a las } \\
\text { Políticas } \\
\text { Públicas }\end{array}$ & $\%$ & $\begin{array}{c}\text { Incremento } \\
\text { del } \\
\text { Conflicto }\end{array}$ & $\%$ \\
\hline $\begin{array}{l}\text { Proponer la } \\
\text { creación de una } \\
\text { circunscripción } \\
\text { de paz. }\end{array}$ & 38,46 & $\begin{array}{l}\text { Reconocimiento } \\
\text { de las } \\
\text { comunidades } \\
\text { indígenas. }\end{array}$ & 38,09 & $\begin{array}{c}\text { Lucha por los } \\
\text { derechos indígenas. }\end{array}$ & 40,0 & $\begin{array}{l}\text { Manejo } \\
\text { indebido de } \\
\text { los recursos } \\
\text { por parte del } \\
\text { Estado. }\end{array}$ & 53,3 & $\begin{array}{c}\text { Incapacidad } \\
\text { de terminar } \\
\text { el conflicto } \\
\text { armado. }\end{array}$ & 46,6 \\
\hline $\begin{array}{l}\text { Humanización de } \\
\text { la guerra }\end{array}$ & 26,92 & $\begin{array}{l}\text { Autonomía } \\
\text { indígena. }\end{array}$ & 28,57 & $\begin{array}{l}\text { La ley contra los } \\
\text { indígenas. }\end{array}$ & 28,0 & $\begin{array}{l}\text { Diferencias } \\
\text { étnicas y } \\
\text { culturales. }\end{array}$ & 26,6 & $\begin{array}{c}\text { Incremento } \\
\text { de la guerra a } \\
\text { causa de las } \\
\text { FARC. }\end{array}$ & 20,0 \\
\hline $\begin{array}{l}\text { Promover la } \\
\text { realización } \\
\text { de diálogos } \\
\text { humanitarios } \\
\text { con los actores } \\
\text { armados. }\end{array}$ & 23,07 & $\begin{array}{l}\text { Violación de } \\
\text { los derechos } \\
\text { humanos y del } \\
\text { territorio. }\end{array}$ & 19,04 & $\begin{array}{l}\text { La formación de una } \\
\text { sociedad más justa. }\end{array}$ & 22,0 & Otros. & 20,0 & Otros. & 33,3 \\
\hline $\begin{array}{l}\text { Cambio de la } \\
\text { cultura política } \\
\text { de los sectores } \\
\text { involucrados. }\end{array}$ & 17,03 & Otros. & 14,28 & $\begin{array}{c}\text { Territorio, } \\
\text { autonomía y cultura } \\
\text { como factores de } \\
\text { disputa política y } \\
\text { militar. }\end{array}$ & 10,0 & & & & \\
\hline
\end{tabular}

Tabla 1. Resultados de Análisis por temas y macro estructuras.

\subsection{Análisis de las microestructuras léxico-semánticas.}

El modelo de gramática, semántica formal, describe las proposiciones ${ }^{6}$ y sus relaciones en la construcción del sentido del discurso o el texto. Según esta perspectiva teórica, el significado (o los significados) de un texto se puede establecer en dos niveles estructurales; el nivel local o microestructural y el nivel global o macroestructural.

6 Se hace alusión al significado de las oraciones. 
La microestructura o micronivel describe la organización de los conjuntos de proposiciones $^{7}$ y las relaciones de correspondencia y coherencia entre ellas además de la función de las categorías implícitas. La macroestructura cumple la misma función descriptiva, pero a un nivel global o macro. En la microestructura o nivel de la oración, que es conformada a su vez por el nivel lexical, se marca desde el principio las relaciones implicadas que delimitan la manera en que una proposición está influida o determinada por una serie de proposiciones que aparecen de forma previa en el texto. Por otra parte, el principio de la coherencia tanto a nivel local o global es lo que permite que un texto tenga sentido.

Sin una coherencia entre las proposiciones y los hechos que denotan (referente), es imposible comprender o producir los significados de los textos o discursos. El sentido de un texto no solo depende de que las proposiciones del mismo nivel sean coherentes entre sí, sino que también establezcan una coherencia entre los dos niveles semánticos o de significado. Van Dijk, (1990, p. 47) señala, además, que la relación de interdependencia entre ambos niveles se apoya en "el principio semántico básico según el cual el sentido del ‘todo' debe especificarse en términos de los significados de sus 'partes”' (p. 45).

La relación entre los niveles se establece mediante el uso de reglas abstractas; el significado y la coherencia global de un texto se derivan de los sentidos o significados locales (secuencias de proposiciones) del discurso. La coherencia entre las proposiciones es de naturaleza funcional. Esto significa que una proposición tiene una función concreta (generalización, especificación, ilustración, contraste) en relación con una proposición que la antecede en el texto (Van Dijk, 1997); la coherencia funcional no solo se encuentra en los niveles locales del texto, sino también en la relación que establecen las proposiciones globales con las proposiciones locales.

Para profundizar la relación entre proposiciones en el texto o discurso, se realiza un análisis léxico-semántico para ver cómo se designa a algunos participantes en el discurso escogido como corpus ${ }^{8}$, de tal forma que se pueda establecer algún tipo de patrón en la elección del uso de cada uno de los términos que se emplean a nivel proposicional y así examinar los posibles efectos de dicha elección. Para esto, se aborda un análisis cuantitativo de todas las formas en las que el discurso se refiere a las personas y estamentos en el corpus seleccionado.

7 Conjunto de significados en las oraciones.

8 Hace referencia al corpus analizado para esta investigación, este constó de dos comunicados de prensa (Declaración del CRIC a los 40 años de su constitución: La María, febrero de 2011 y el Pronunciamiento del CRIC- Toribío, 20 de julio de 2011, "terminar la guerra, defender la autonomía, reconstruir los bienes civiles y construir la paz"), además del discurso de apertura del constituyente Alfonso Peña Chepe en la constituyente de 1991, publicado en la Gaceta constitucional N 67. 
En ese análisis cuantitativo, se toma en cuenta la temática en que cada término aparece en cada proposición y el nivel de coherencia que adquiere esta, dependiendo de las terminologías usadas y así poder determinar si esta elección de términos sigue algún tipo de patrón y si es coherente con lo planteado en el discurso.

Por otro lado, se analizan los significados que se expresan explícitamente y se revela cuáles permanecen implícitos y/o se dan por supuestos para comprender el papel de la ideología en la selección de aquellos que subyacen. Así se muestra cómo la ideología condiciona la información que se presenta de manera explícita y de manera implícita, y si la elección obedece a estrategias ideológicas de autopresentación positiva o negativa, y de qué formas son usadas como estrategias de persuasión.

\subsection{La estructura léxico-semántica}

La coherencia de un texto está dada por la interrelación semántica que se encuentre en él y que forma la estructura global del significado en un texto. Se puede considerar cada uno de estos puntos como formados por pequeñas unidades temáticas, es decir, por minúsculas categorías sémicas. De este modo, se va dando lugar a un sistema que se va tejiendo a partir de la reiteración de elementos similares o compatibles, como los nudos de un tejido. La complejidad de la narración podría verse, entonces, en la medida en que se da una mayor cantidad de nudos en las unidades temáticas paralelas o complementarias, 0 bien disyuntivas u opuestas, a través de lo que se ha llamado cohesión léxica.

Desde este punto, se hace necesario iniciar, para el análisis léxico semántico, a partir de la coherencia en el texto sobre diversas temáticas expuestas en el discurso. Así que el análisis exhaustivo de estos sistemas, permitirá comprender de qué modo opera la ideología sobre su producción discursiva en los planteamientos políticos e ideológicos del CRIC, representados por el constituyente Alfonso Peña Chepe. Por otro lado, el estudio de estas microestrategias utilizadas en su discurso ayudará a caracterizar mejor determinadas perspectivas y juicos de valores, que surgen del discurso de legitimización.

Como estrategia para este fin, se eligió la división temática y macro proposicional, debido a que cada macro proposición se deriva de una serie de microproposiciones concretas en el texto y es en estas microproposiciones donde se sucederá el microanálisis de las principales estrategias retórico-argumentativas a las que el CRIC recurre cuando habla sobre legitimización. Esta división temática obedece al hecho de que, dependiendo de los distintos temas tratados, la argumentación y la retórica suelen ser de algún modo diferentes, ya que el contenido semántico de cada uno de los temas determina el contenido y la forma de los argumentos. 
Por lo anteriormente mencionado, se hace necesario estudiar por separado cada una de las macroproposiciones, de manera que salgan a la luz las distintas temáticas como paso preliminar para establecer posibles patrones sobre el uso de las estrategias retórico argumentativas utilizadas con mayor frecuencia para legitimar sus acciones políticas, sus opiniones y sus actitudes. En suma, para llevar a cabo este análisis, se indaga en cada macroproposición aquellas estrategias retórico argumentativas que aparecen a nivel micro de manera más reiterada.

\subsection{La topología del discurso.}

La teoría y métodos que corresponden a la "Topología del discurso" han venido siendo desarrollados a través de los años por el profesor Antonio Rodríguez de las Heras", método que se ha venido aplicando para un análisis lexical de los contenidos proposicionales y lexicales en el texto. Para conocer los fundamentos teóricos y metodológicos de este método, es obligatorio dirigirse a los trabajos de este investigador.

Sin embargo, por el tipo de análisis discursivo que se presenta aquí, es necesario describir superficialmente sus postulados, con el fin de ampliar las características de análisis indispensables para un discurso de un fuerte contenido ideológico, marcado en el léxico utilizado por Alfonso Peña Chepe en la apertura de las comisiones que modificarían la constitución colombiana de 1880, además de los comunicados de prensa del CRIC utilizados para este estudio.

Este método afirma que sí se puede describir y caracterizar una ideología. El método utilizado por el profesor Rodríguez de las Heras, intenta explicar por qué una ideología no es solo una colección de proposiciones, sino, a su vez, una formulación (afirmativa 0 negativa) de esas proposiciones (ideas y valores) dados en el discurso. Una formulación (relaciones de coalición y de antagonismo) que se basa en una reglamentación (una especie de gramática del pensamiento).

Las relaciones de coalición y de antagonismo pueden representarse gráficamente. La coalición (la afirmación) se representa con el símbolo de (=) uniendo los conceptos en forma de tríadas. Sobre la explicación de este tipo de análisis se profundizará más adelante.

9 Antonio Rodríguez de las Heras, catedrático de Historia Contemporánea e Historia del Tiempo Presente de la Universidad Carlos III de Madrid. 


\subsection{Fundamentos teóricos de la topología del discurso}

Una ideología no es una recopilación de proposiciones, en sí consiste, como lo había mencionado anteriormente, en una formulación (afirmativa o negativa) de esas proposiciones (ideas y valores). La coalición (la afirmación) se representan con el símbolo de (=) uniendo los conceptos o ideas en tríos significativos:

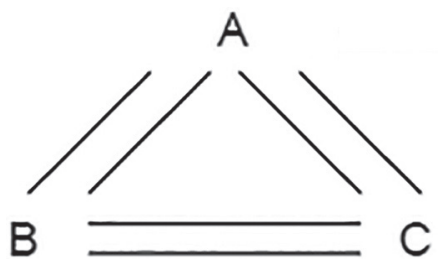

Figura 1.

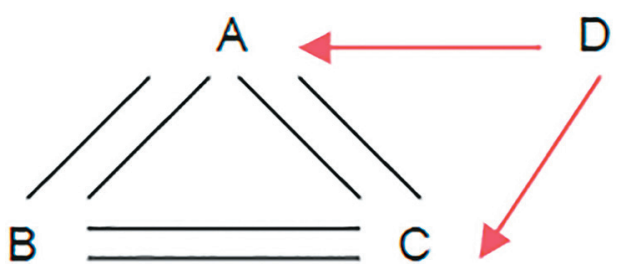

Figura 2.

Fuente: http://earchivo.uc3m.es/bitstream/handle/10016/4944/TESIS\%20DOCTORAL\%20JCPQ. pdf;jsessionid=B17A7762FBD4AFE725BD331D3EBC147A?sequence $=1$

Por otro lado, la diferenciación o relación antagonista (la negación) es representada por el símbolo de $(\uparrow)$. La diferenciación se une también para conformar un nuevo constructo significativo en relación con el antagonismo (ver figura 2).

Es así como la unión de los conceptos toman relación entre ellos mismos para conformar una "ley de composición" que muestra relaciones no expresadas o para advertir sobre planteamientos débiles 0 inestables ${ }^{10}$.

Tabla 2. Ley de composición

$$
\begin{array}{ll}
=\mathrm{y}=\text { resulta }= & \text { coalición y coalición resulta coalición } \\
=\mathrm{y} \uparrow \text { resulta } \uparrow & \text { coalición y antagonismo resulta antagonismo } \\
\uparrow \mathrm{y}=\text { resulta } \uparrow & \text { antagonismo y coalición resulta antagonismo } \\
\uparrow y \uparrow \text { resulta }= & \text { antagonismo y antagonismo resulta coalición }
\end{array}
$$

Fuente: http://earchivo.uc3m.es/bitstream/handle/10016/4944/TESIS\%20DOCTORAL\%20JCPQ. pdf;jsessionid=B17A7762FBD4AFE725BD331D3EBC147A?sequence $=$

10 Esto lo aclara Julio César Puente Quintanilla, en su tesis doctoral "Análisis de los discursos de los Jefes de Estado y de Gobierno en las Cumbres Iberoamericanas de Naciones" p. 26. 


\subsection{La topología del discurso}

La metodología desarrollada por Rodríguez de las Heras, conocida como topología del discurso, se basa fundamentalmente en que todo pensamiento se entrecruza en distintas redes de conceptos relacionados entre sí. Esta red al concretarse, forma las ideologías del hablante. Este método es compuesto por un número de reglas o elementos llamados de COMPOSICIÓN y de TRANSFORMACIÓN.

La estructura ideológica, para adquirir la condición de tal, ha de contar también con todos y cada uno de estos componentes:

1. Un conjunto de elementos: [conceptos $(*)$, flecha $(\Longrightarrow)$ y doble barra $(=)$.

2. Unas reglas de formación o composición. No se puede presentar de modo aislado ningún elemento (ni un concepto, ni una flecha, ni una doble barra). Al menos tiene que haber dos conceptos y, entre ellos, una flecha o una doble barra.

La flecha entre dos conceptos supone una relación de oposición entre ellos. Un concepto niega a otro en esa ideología. La doble barra supone una relación de complementariedad entre conceptos, un concepto no se desliga del otro. Una ley de transformación no es otra cosa que la lógica interna que descubrimos en cualquier ideología. Ante tres conceptos, si se conocen dos de sus relaciones, la tercera viene determinada por estas dos anteriores.

\subsection{Microestrategias léxicas}

En la tabla tres (3) se observa la categorización por porcentajes de aparición de los términos más comunes en los discursos seleccionados para el estudio, haciendo igualmente una agrupación por relaciones semánticas entre las palabras en su orden de aparición.

Tabla 3. Categorización por porcentajes de aparición de los términos más comunes.

Elaboración propia

\begin{tabular}{|l|c|l|}
\hline \multicolumn{1}{|c|}{ PALABRA } & $\begin{array}{c}\text { PORCENTAJE } \\
\text { PONDERADO \% }\end{array}$ & \multicolumn{1}{|c|}{ PALABRAS SIMILARES } \\
\hline Población & 1.01 & $\begin{array}{l}\text { Población, pueblo, pueblos, civil, civiles, cultural, popular, } \\
\text { populares }\end{array}$ \\
\hline Indígenas & 0,81 & Indígena, Indígenas \\
\hline Armado & 0,71 & Armado, armados, arma, armas \\
\hline Territorio & 0,67 & Territorio, territorios, tierra, tierras \\
\hline Paz & 0,61 & Paz \\
\hline
\end{tabular}




\begin{tabular}{|l|c|l|}
\hline Guerra & 0,60 & Guerra, guerras \\
\hline Política & 0,46 & Política, políticas \\
\hline Derechos & 0,42 & Derecho, derechos \\
\hline Comunidades & 0,40 & Comunidad, comunidades \\
\hline Gobierno & 0,40 & Gobierno, gobiernos \\
\hline Conflicto & 0,39 & Conflicto, conflictos \\
\hline Guerrilla & 0,38 & Guerrilla, guerrillas, irregular, insurgencia. \\
\hline Actores & 0,38 & Actor, actores, principal \\
\hline Político & 0,37 & Político, políticos \\
\hline Militares & 0,35 & Militar, militares \\
\hline Solución & 0,35 & Solución \\
\hline Estado & 0,31 & Estado, estados \\
\hline Autonomía & 0,25 & Autonomía \\
\hline
\end{tabular}

De este resultado, entre los términos más comunes en los textos estudiados, se hizo una selección de los de mayor trascendencia para el estudio. Para esto se seleccionaron 18 palabras, según su orden de aparición e importancia significativa. Cabe anotar, que la elección se hizo solo tomando los sustantivos, ya que parte de este estudio busca enfocarse en la nominalización de los actores en el texto estudiado (véase figura 3).

Figura 3. Constructo conglomerado de discursos por jerarquía

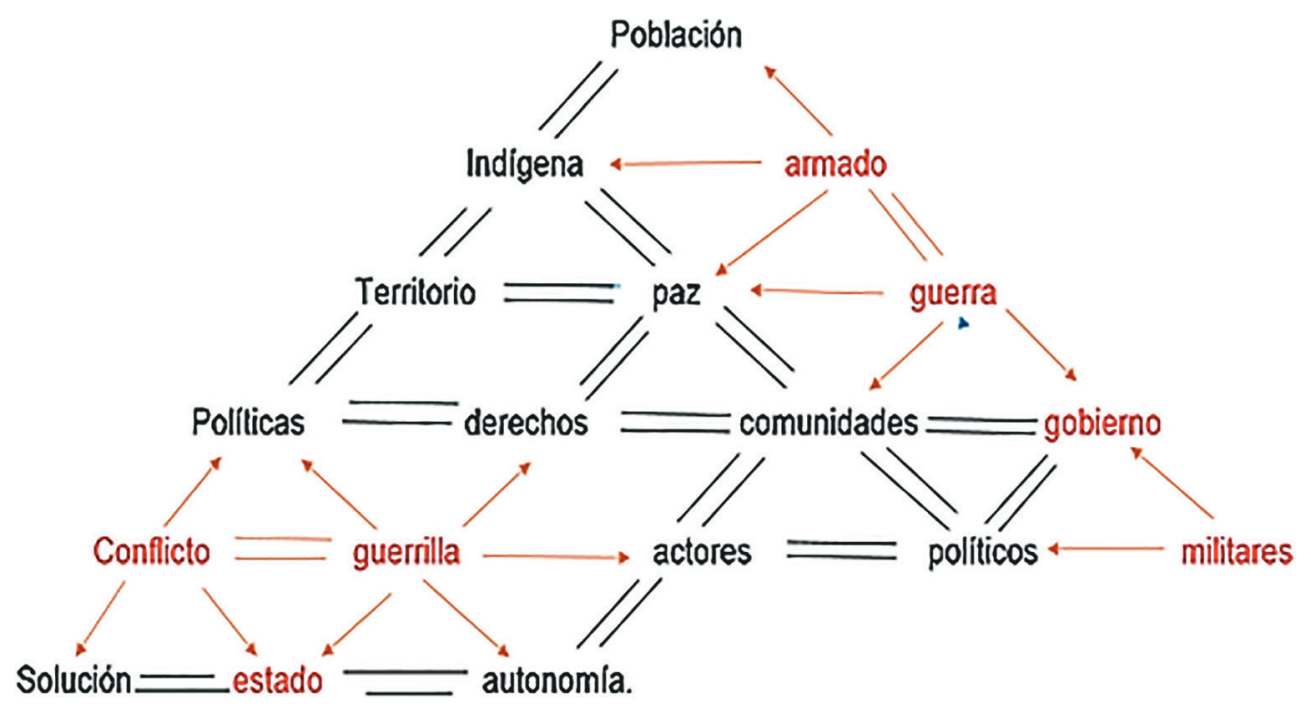

Fuente: elaboración propia. 


\subsection{Síntesis y análisis de las microestrategias léxico-semánticas}

El conglomerado de los discursos analizados, que corresponden a las propuestas y expectativas políticas de la comunidad del Cauca, en comparación y teniendo en cuenta sus significados próximos o similares (ver tabla 3), arrojan los siguientes resultados:

En primera instancia, para este grupo indígena resulta también prioritario atender los temas que correspondan a la protección de la "población" "indígena" y del "territorio", hacia la "paz", con unas "políticas" de "derecho" que colaboren a las "comunidades" en busca de su "autonomía", con hechos "políticos" que llevarían a la finalización del conflicto armado.

En segunda instancia, encontramos como referentes antagónicos todos los que hacen alusión directa a la continuación del conflicto como: "armas o armamento", que conllevaría a la perpetuación de la "guerra", por medio del mal manejo del "gobierno" y sus fuerzas "militares", sumándose así a la prolongación del "conflicto" con la "guerrilla" y otros "actores" sin que el "estado" pueda dar solución a esto.

Como lo hemos visto anteriormente, los valores positivos se encuentran enmarcados en la temática principal de este estudio que es "el fin del conflicto armado" y como conceptos antagónicos y la contratesis encontrada en los resultados de este análisis que sería "la perpetuación del conflicto armado en Colombia". Es así como se puede establecer una relación de coherencia entre conceptos y temáticas en el discurso.

\section{Conclusiones}

Para la obtención de estos resultados, se trazaron tres objetivos específicos que, en conjunto, ofrecieron las herramientas necesarias para alcanzar el propósito central. Con el primero de ellos se pretendía analizar los recursos lingüísticos presentes en el discurso de inclusión y reconocimiento indígena; con el segundo, caracterizar y clasificar las macroestrategias, así como las microestrategias léxico-semánticas utilizadas en el discurso político de algunos líderes indígenas en Colombia. Con el tercero, determinar los temas más recurrentes en la argumentación política de las comunidades indígenas del Cauca y cómo estos pueden determinar una ideología en su discurso.

Para la consecución del primer objetivo cabe anotar que en el análisis desarrollado para establecer el índice de palabras más comunes en el texto, se encontró que es muy frecuente el uso de la adjetivación como recurso retórico dentro del discurso político del CRIC. Ateniéndonos a la definición de adjetivo y su función según Bello. Son palabras que modifican directamente al sustantivo. 
Con respecto al segundo objetivo planteado, se puede concluir que la clasificación de las macroestrategias y microestrategias léxico-semánticas, ha llevado a determinar que el tema de la "finalización del conflicto armado" en Colombia se ha convertido en la temática primordial por tratar, por encima de temas como: defensa de la autonomía, incremento del conflicto armado, manejo de las políticas públicas y el del reconocimiento de las comunidades indígenas. Esto lleva a categorizar las prioridades actuales de los representantes de las comunidades indígenas del Cauca, las cuales como se ve en sus discursos, sin la finalización del conflicto armado es muy improbable que se pueda constituir un proyecto de inclusión y reconocimiento a nivel nacional.

Por otra parte, el análisis de las microestructuras léxico-semánticas ayuda a determinar los factores antagónicos y de coalición permanentes en los discursos políticos de las comunidades indígenas del Cauca, dando como resultado que los conceptos positivos o de coalición son los más significativos en su discurso y los antagónicos, los que plantean la antítesis en su discurso.

Otro factor para tener en cuenta entre los resultados, es el planteamiento de la siguiente tesis: "La urgencia de finalizar el conflicto armado en Colombia" y el descubrimiento de su antítesis a partir de los factores negativos o antagónicos que sería "La perpetuación del conflicto armado en Colombia".

Ya para finalizar, el análisis temático y macro-estructural sumado al análisis de las microestrategias léxico semánticas en el discurso de los líderes de la comunidad indígena del Cauca, nos lleva a determinar algunos factores ideológicos, como dice van Dijk: "La implicación en el discurso en particular, no presupone únicamente representaciones individuales, tales como modelos (experiencias, planes); también exigen representaciones que son compartidas por un grupo o una cultura, como el conocimiento, las actitudes y las ideologías"11, es por esto que podemos hacer alguna interpretación a partir de los resultados sobre la ideología de esta comunidad.

Estas representaciones compartidas, como lo dice Van Dijk (1999), se definen en esta comunidad como la no participación en el conflicto armado, pero atendiendo siempre a las movilizaciones sociales de carácter pacífico, no siendo partícipes tampoco ni colaboradores de ningún grupo sea del ejército nacional o de los de insurgencia armada, esto en la búsqueda de su autonomía política y el resguardo de territorios tradicionales. Además, se ha visto que esta comunidad es partidaria del fin del conflicto armado y ha aportado con propuestas concretas soluciones a la finalización del mismo.

11 Representaciones socio-mentales. El análisis crítico del discurso (Teun A. van Dijk. En: Anthropos, Barcelona), 186, septiembreoctubre 1999, pp. 26. 
Es por esto entonces, que no podemos rotular a estas comunidades como de "izquierda" o de "derecha", ya que en la búsqueda de su autonomía quieren establecer propuestas y planteamientos propios de su transcurrir histórico y cultural, según dictan sus tradiciones. Asimismo, no incurrir en el error de verlas como parte del problema sino, al contrario, escuchar y sopesar sus propuestas como parte de una posible solución a la violencia que sufre el territorio colombiano.

\section{Referencias bibliográficas}

Consejo Regional Indígena del Cauca. (1976). Cinco años del CRIC. Bogotá. en: Unidad Indígena $\mathrm{n}^{0} 11$, febrero. Onic.

Foucault, M. (1970). El orden del discurso. Traducción de Alberto González Troyano. Buenos Aires: Tusquets Editores.

ONIC y Consejo indígena de paz. (1985). Resolución del Resguardo de Vitoncó, Cauca, en: Los indígenas y la paz: pronunciamientos, resoluciones, declaraciones y otros documentos de los pueblos y organizaciones indígenas sobre la violencia armada en sus territorios, la búsqueda de la paz, la autonomía y la resistencia. Bogotá: Organización nacional Indígena de Colombia, Arfo Editores e impresores.

Schelotto, M. (2009). Aplicación de un método de análisis del discurso: Cartas desde el cautiverio del General Líber Seregni. Madrid: Universidad Carlos iii.

Van Dijk, T. (1990). El análisis crítico del discurso. Barcelona: Gedisa.

Van Dijk, T. (2003). Dominación étnica y racismo discursivo en España y América Latina. Barcelona: Gedisa.

Van Dijk, T. (1999). Ideología. Una aproximación multidisciplinaria. Barcelona: Gedisa.

Van Dijk, T. (2009). Discurso y poder. Barcelona: Gedisa. 